\title{
Battlefield Advanced Trauma Life Support
}

\section{CHAPTER 8 HEAD INJURIES}

\section{Aim}

0801. On successfully completing this topic you will be able to:

- Discuss general management of the unconscious casualty with a head injury.

- Understand the anatomy and pathophysiology of head injury.

- Understand than an altered level of consciousness is the hallmark of brain injury.

- Demonstrate the initial assessment and management of a casualty with a head injury.

- Assess the criteria for neurosurgical referral in war.

\section{Introduction}

0802. Head injury is common: it carries a high mortality both in peacetime and on the battlefield. The aim of initial management of a casualty with a head injury is:

\section{To prevent secondary brain injury due to cerebral hypoxia \\ 2. To identify injuries needing urgent surgery.}

0803. This is done by appropriate management of: Airway, Breathing, Circulation, and D - repeated assessment for neurological DEFICIT.

\section{Anatomy and Pathophysiology}

0804. The skull is a rigid box containing brain, cerebrospinal fluid (CSF) and blood vessels:

- The brain is surrounded by a series of membranes. The outer, thick membrane is called the dura. Beneath the dura is a more flimsy membrane called the arachnoid. The CSF flows in the space deep to the arachnoid. Bleeds are described as occurring outside or inside these membranes hence extradural, subdural and subarachnoid.

- Blood is supplied to the brain by the internal carotid and vertebral arteries and eventually drains via the internal jugular veins.

- The brain is very active and needs a lot of oxygen and substrate to survive. Loss of oxygenated blood flow for more than three minutes will cause brain damage.

- Intracranial pressure (ICP) is the pressure inside the skull. Because the skull is a rigid box, an increase in the size of the brain, the volume of blood or amount of cerebrospinal fluid within it, will cause an increase in ICP. Cerebral perfusion refers to the supply of oxygenated blood to the brain.

- Cerebral perfusion pressure (CPP) depends on the blood pressure pushing blood into the brain and the resistance to this blood flow from the ICP. If the ICP rises then a higher blood pressure is needed to supply blood to the brain. In other words -

$$
\text { CPP = MAP - ICP }
$$

Note: MAP (Mean Arterial Pressure) ${ }^{1}$. Normal value 70 to $90 \mathrm{mmHg}$.

Normal ICP $10 \mathrm{mmHg}$

If CPP is less than $50 \mathrm{mmHg}$, cerebral hypoxia will follow.

- Lack of oxygen (hypoxia) makes the brain swell. Raised carbon dioxide levels in the blood causes cerebral blood vessels to dilate which increases the volume of blood in the head and further raises ICP. This is why management of Airway and Breathing is essential.

- Raised ICP may also be due to obstruction of venous drainage from the head.

This may be due to:

- Pressure on the neck veins.

- Increased pressure inside the chest.

0805. Rising ICP, brain swelling or expanding haematomas inside the head can cause a variety of neurological signs:

- Pressure of the third cranial nerve (oculomotor) will result in a dilated pupil on the same side as the injury.

- Damage to the motor or sensory cortex (or tracts leading from them) will result in a motor or sensory DEFICIT on the contralateral side to the injury.

- A decrease in level of consciousness is the marker of brain injury. Generally, the more deeply unconscious a casualty becomes, the more serious is the injury. Coma is a term used for unconsciousness although to be accurate it should only be used for deeply unconscious casualties (see paragraph 0829).

- Pressure on the lower part of the brain (brain stem) where the respiratory and 
cardiovascular centres lie, produces respiratory or cardiovascular abnormalities (change in heart rate and blood pressure, change in breathing pattern and rate).

0806. It follows that preventing a rise in intracranial pressure or a fall in cerebral perfusion is vital in the overall management of head injury. This must never be forgotten.

\section{Brain Injury}

0807. Primary brain injury is the neurological damage produced by the traumatic event, for example, a blow to the head or damage from a gunshot wound. Secondary brain injury is the neurological damage produced by what follows on from the traumatic event. Causes include: hypoxia, reduced cerebral perfusion, raised ICP, convulsions and infection.

\section{Primary Brain Injury}

0808. Primary brain injury may be diffuse or focal.

\section{Diffuse Brain Injury}

0809. Blunt injury to the brain may cause diffuse brain injury, particularly when rapid head motion (acceleration or deceleration) leads to widespread damage within the brain substance. Such injuries form a spectrum extending from mild confusion to severe injury.

\section{Concussion}

0810. Concussion is a brain injury accompanied by a brief loss of consciousness and, in its mildest form, may cause only temporary confusion or amnesia. With mild forms of concussion, most casualties will be slightly confused and may be able to describe how the injury occurred. They are likely to complain of mild headache, dizziness or nausea. The mini-neurological examination will not show localising signs. With more severe concussion there is a longer period of unconsciousness, longer amnesia (for time both before and after the injury) and there may be focal signs. The duration of amnesia needs to be recorded.

\section{Diffuse axonal injuries}

0811. At the other end of the spectrum from mild concussion, diffuse axonal injury is so severe as to cause a characteristically long coma, present in $44 \%$ of cases. The overall mortality rate is over $30 \%$, rising to $50 \%$ in its most severe form. The treatment of such injury involves prolonged controlled ventilation in an intensive care unit, a facility that may not be available. Because the mortality is so high, these casualties have a low priority for evacuation.

\section{Focal Brain Injuries}

0812. Brain injuries that produce a contusion or haematoma in a relatively small area of the brain are amenable to emergency surgery. These conditions must be diagnosed early as rapid evacuation to a surgical or neurosurgical unit for early surgery, greatly reduces the morbidity and mortality.

\section{Contusions}

0813. These are caused by blunt injury producing acceleration and deceleration forces on the brain tissue resulting in tearing of the small blood vessels inside the brain. Contusions can occur immediately beneath the area of impact when they are known as coup injuries, or at a point distant from the area of impact in the direction of the applied force when they are known as contrecoup injuries. If the contusion occurs near the sensory or motor areas of the brain, these casualties will present with a neurological deficit. Precise diagnosis requires appropriate imaging (CT scanning), consequently, the treatment is supportive, aimed at the avoidance of secondary brain injury.

\section{Intracranial haemorrhage}

0814. Haemorrhage may arise either from meningeal vessels or from vessels within the brain substance.

\section{Extradural haemorrhage}

0815. This is caused by a tear in a dural artery, most commonly the middle meningeal artery. This can be torn by a linear fracture crossing the temporal or parietal bone and injuring the artery lying in a groove on the deep aspect of the bone (see Fig 8.1). Isolated extradural haemorrhage is unusual, accounting for only $0.5 \%$ of all head injuries and less than $1 \%$ of injuries causing coma. The importance of early recognition of this injury lies in the fact that, when treated appropriately, the prognosis is good because of the lack of underlying serious injury to brain tissue. If missed, the rapidly expanding haematoma causes ICP to rise, reducing cerebral perfusion and leading to cerebral hypoxia, coma and death.

0816. The typical symptoms and signs of extradural haemorrhage are:

- Loss of consciousness followed by a lucid interval (which may not be a complete return to consciousness).

- Secondary depression of consciousness.

- Dilated pupil on the side of injury.

- Weakness of the arm and leg on the contralateral side to the injury.

\section{Subdural haematoma}

0817. This is more common than extradural haemorrhage and is found in 30\% of all severe head injuries. The mortality rate is up to $60 \%$ because, in addition to the compression caused by the subdural clot, there is often major injury to the underlying brain tissue. The haematoma can arise from 


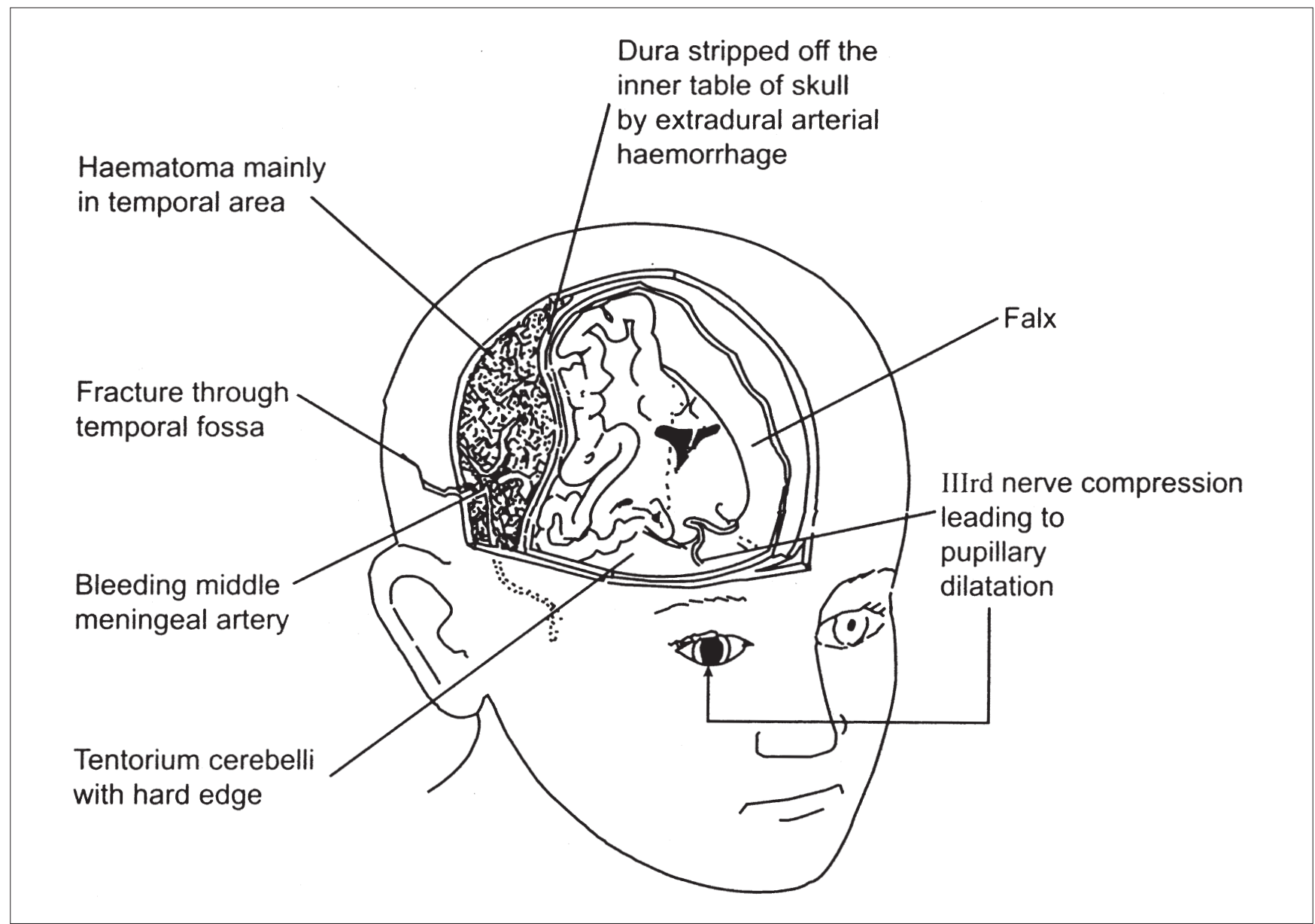

Fig 8.1 Acute extradural haematoma

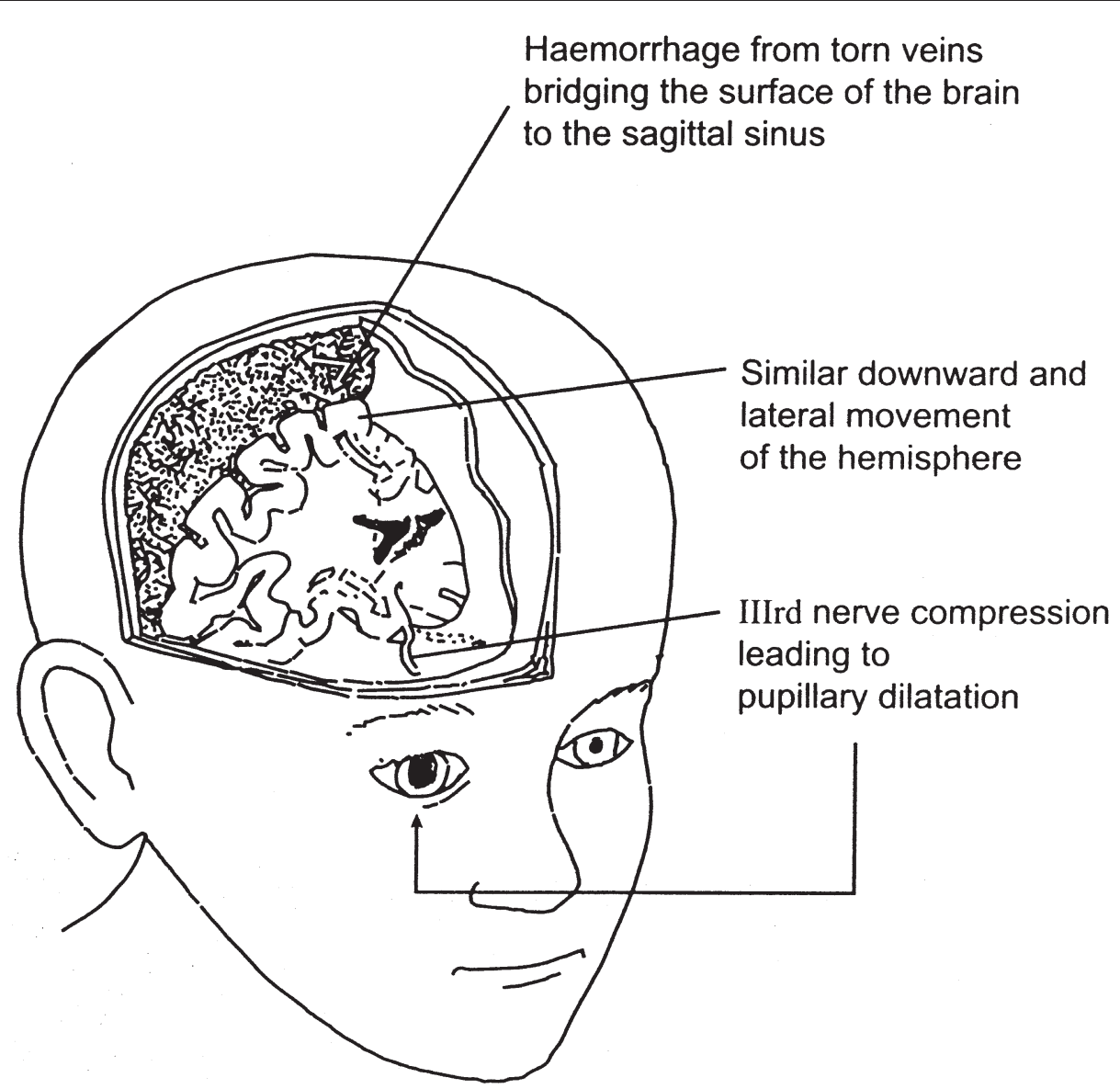


tears in the bridging veins between the cortex and the dura or from laceration of the brain substance and the cortical arteries (see Fig 8.2). Levels of consciousness will vary depending on the underlying brain damage and rate of haematoma formation. The pupil on the affected side will become dilated together with contralateral limb weakness.

\section{Subarachnoid haemorrhage}

0818. Where haemorrhage has occurred into the subarachnoid space, the irritant effect of the bloody cerebrospinal fluid causes headache, photophobia and neck stiffness. On its own this is not serious, but prognosis is poor if associated with a more severe head injury.

\section{Intracerebral laceration}

0819. These can be caused by:

- Impalement injury. All foreign bodies found protruding from the skull must be left in place; these will be removed at the neurosurgical unit. Skull X-rays will show the angle and depth of penetration. Care must be taken during evacuation to ensure there is no further penetration.

- Gunshot wounds and fragment wounds. Prognosis is determined by the size of missile and energy transfer, the number of penetrating fragments and the length of the wound track. Casualties with these injuries who are in coma have a very high mortality rate. (See Table 8.1).

Table 8.1 Mortality rates - penetrating head injuries

\begin{tabular}{|c|c|c|}
\hline $\begin{array}{c}\text { Level of } \\
\text { Consciousness }\end{array}$ & $\begin{array}{c}\text { Percentage } \\
\text { Mortality }\end{array}$ & $\begin{array}{c}\text { Approximates } \\
\text { to: }\end{array}$ \\
\hline Alert & 11.5 & $\mathrm{~A}$ \\
\hline Drowsy & 33.3 & $\mathrm{~V}$ \\
\hline Reaction to pain & 79.1 & $\mathrm{P}$ \\
\hline Coma & 100 & $\mathrm{U}$ \\
\hline
\end{tabular}

Note: Through-and-through injuries, sideto-side injuries and injuries in the lower region of the brain stem, all have a poor outcome. All these factors must be considered when deciding the priority for evacuation. Open brain injury in a conscious casualty carries a good prognosis if surgery is not delayed. Scalp haemorrhage should be stopped, entrance and exit wounds covered with sterile dressings and the casualty evacuated to a neurosurgical unit.

\section{Assessment of Head Injury History}

0820. Knowing the mechanism of injury provides important information for both prognosis and early selection for evacuation to a neurosurgical unit. For example, a fall from a height, as opposed to involvement in a road traffic accident, quadruples the likelihood of an intracranial haematoma. With gunshot and fragment injuries to the head, the initial level of consciousness is related to mortality.

\section{Primary Survey}

0821. This follows the BATLS A B C D E protocols. For head injury understand:

\section{A. Airway.}

A casualty with a reduced level of consciousness is likely to have a compromised airway.

\section{B. Breathing.}

Good ventilation ensures the brain receives blood containing enough oxygen and not excess carbon dioxide.

Raised intrathoracic pressure (as happens in tension pneumothorax) will interfere with venous drainage from the head and raise ICP.

\section{Circulation.}

Never presume the brain injury is the cause of hypotension. Scalp lacerations may bleed profusely but hypotension secondary to an isolated brain injury is uncommon and usually fatal.

\section{Always presume that hypotension is due to hypovolaemia not brain injury and look for a source of blood loss elsewhere.}

Cushing's response (progressive hypertension, bradycardia and slowing of respiratory rate) is an acute and potentially lethal response to rapidly rising intracranial pressure. This usually indicates a need for immediate surgery or precedes death.

\section{Disability.}

A rapid assessment of conscious level is made in the primary survey using AVPU, that is, is the casualty Alert or responding to Voice or only responding to Pain or Unresponsive?

\section{SECONDARY SURVEY}

\section{Mini-neurological examination}

0822. In the secondary survey, the minineurological examination is carried out to:

- Identify neurological injuries.

- Establish anatomical diagnosis.

- Identify casualties needing early evacuation for surgery.

0823. The mini-neurological examination assesses:

- Pupillary Function.

- Lateralised limb weakness.

- Level of consciousness by the Glasgow Coma Scale.

0824. The mini-neurological examination serves to determine the severity of the brain injury. When applied repeatedly at various points in the evacuation chain, it can be used to determine objectively any neurological deterioration. Remember: 


\section{Alteration of the level of \\ consciousness is the hallmark of brain injury.}

\section{Mini-neurological examination - pupils}

0825. Evaluate the pupils for their equality and response to bright light. A difference in diameter of the pupils of more than $1 \mathrm{~mm}$ is abnormal, but remember that a local injury to the eye may be responsible for this abnormality. Normal reaction to a bright light is brisk constriction of the pupil; a more sluggish response may indicate brain injury. A dilated pupil on the same side as the injury indicates compression of the brain on that side.

\section{Mini-neurological examination - extremity movement}

0826. Observe the spontaneous limb movements for equality; if movement is negligible then you must assess the response to a painful stimulus ${ }^{1}$. Any delay in onset of movement, or late realization of movement following a painful stimulus, is significant. Obvious limb weakness localized to one side suggests an intracranial injury causing brain compression on the opposite side.

\section{Mini-neurological examination - Glasgow Coma Scale}

0827. This provides a quantitative assessment of the level of consciousness. It is the sum of scores awarded for three types of response:

- Eye opening (E). The scoring of eye opening is not possible if the eyes are so swollen as to be permanently shut. This fact must be documented.

\begin{tabular}{|l|l|}
\hline $\begin{array}{l}\text { Spontaneous, that is, open with normal } \\
\text { blinking. }\end{array}$ & 4 points \\
\hline Eye opening to speech on request. & 3 points \\
\hline Eye opening only to pain stimulus. & 2 points \\
\hline No eye opening despite stimulation. & 1 point \\
\hline
\end{tabular}

- Verbal response (V). The scoring of verbal response is not possible if the casualty cannot speak because of endotracheal intubation. This fact must be documented.

\begin{tabular}{|l|c|}
\hline $\begin{array}{l}\text { Orientated, spontaneous speech; knows } \\
\text { name, age and so on. }\end{array}$ & 5 points \\
\hline $\begin{array}{l}\text { Confused conversation but answers } \\
\text { questions. }\end{array}$ & 4 points \\
\hline $\begin{array}{l}\text { Inappropriate words, that is, garbled } \\
\text { speech but with recognizable words. }\end{array}$ & 3 points \\
\hline Incomprehensible sounds or grunts. & 2 points \\
\hline No verbal response. & 1 point \\
\hline
\end{tabular}

- Motor response (M). The best response obtained for either of the upper extremities is recorded even though worse responses may be present in other extremities.

\begin{tabular}{|l|c|}
\hline $\begin{array}{l}\text { Obeys commands and moves limbs to } \\
\text { command. }\end{array}$ & 6 points \\
\hline $\begin{array}{l}\text { Localizes, for example, moves upper arm } \\
\text { to pain stimulus on head. }\end{array}$ & 5 points \\
\hline Withdraws from painful stimulus on limb. & 4 points \\
\hline Abnormal flexion or decorticate posture. & 3 points \\
\hline Extensor response, decerebrate posture. & 2 points \\
\hline No movement to any stimulus. & 1 point \\
\hline
\end{tabular}

The Glasgow Coma Scale is scored differently in children - See Supplement No 1.

0828. The Mini-neurological examination can thus be used to determine the severity of brain injury at a particular time. Subsequent reassessment can be used to detect any deterioration. For example, if the Glasgow Coma Scale has decreased by two points or more, deterioration has occurred. A decrease of three points or more is a bad prognostic indicator and demands immediate treatment. Dramatic changes in the Glasgow Coma Scale are often preceded by more subtle signs indicating deterioration, notably:

- A very severe headache or increase in the severity of headache.

- An increase in the size of one pupil or the development of unilateral limb weakness.

Changes in the casualty's
neurological condition can be
detected only if the mini-
neurological examination is
repeated and re-documented at
various points along the evacuation
chain.

\section{All previous efforts will be wasted if the deterioration is not detected, recorded and acted upon.}

\section{Definition of Coma}

0829. Coma can be defined as that state in which:

- There is no eye opening despite stimulus.

- The casualty does not follow commands.

- There is no verbalization.

0830. Consequently the objective Glasgow Coma Scale score that equates to coma is 8 or less. The scale gives some indication of the severity of brain injury as follows:

\begin{tabular}{|cc|}
\hline Score 8 or less & Severe \\
Score 9 to 12 & Moderate \\
Score 13 to 15 & Minor \\
\hline
\end{tabular}

0831. When dealing with a closed head injury you must never assume that coma is due to alcohol or sedative drugs, even if the casualty has had access to them. You must still consider the mechanism of injury in 
addition to the possible pharmaceutical effects of alcohol and drugs.

\section{In BATLS, \\ The unconscious casualty who has \\ been drinking has a head injury until proved otherwise.}

\section{Secondary Survey - Specific Injuries}

\section{Scalp Wounds}

0832. The scalp is arranged in layers. It is highly vascular and a laceration will often result in profuse haemorrhage. The bleeding point should be located and the haemorrhage arrested. This may include the use of haemostatic surgical clips and ligatures, particularly where the laceration is deep. Direct pressure may not be sufficient. The wound should be inspected carefully for signs of skull fracture and irrigated to remove debris and dirt.

0833. Gentle palpation of the scalp wound wearing a sterile glove may enable you to diagnose the presence of a skull fracture. If an open or depressed fracture is detected, close the wound with sutures, apply a dressing, give antibiotics and evacuate the casualty to a neurosurgical unit. Do not remove any bone fragments at this stage.

\section{Skull Fractures}

0834. Although skull fractures are common, many major brain injuries will occur without the skull being fractured and many skull fractures are not associated with severe brain injury. Where the mini-neurological examination identifies the presence of a severe brain injury, time taken to search for a skull fracture should never delay definitive management. The significance of a skull fracture is that it identifies a casualty with a higher probability of having or developing an intracranial haematoma. All casualties with skull fractures should be detained for observation.

- Linear skull fractures. These are particularly important when the fracture crosses the line of intracranial vessels indicating an increased risk of intracranial haemorrhage.

- Depressed skull fractures. All depressed skull fractures should be evacuated for neurosurgical unit assessment; they may be associated with underlying brain injury and require operative elevation to reduce the risk of infection.

- Open skull fractures. By definition, there is direct communication between the outside of the head and brain tissue because the dura covering the surface of the brain is torn. This can be diagnosed if brain tissue is visible on examination of the scalp wound or if cerebrospinal fluid is seen to be leaking from the wound. These fractures all require operative intervention as the risk of infection is high. Give prophylactic antibiotics in accordance with Casualty Treatment Regimes.

- Basal skull fractures. The base of the skull does not run horizontally backwards but diagonally (See Fig 8.3); basal skull fractures will produce signs along this diagonal line. They can be diagnosed clinically in the presence of cerebrospinal fluid leaking from the ear (otorrhoea) or the nose (rhinorrhoea). When cerebrospinal fluid is mixed with blood it may be difficult to detect; the ring sign can be used to detect its presence. Allow a drop of the fluid to fall on a piece of absorbant material, such as filter paper; if cerebrospinal fluid is present the blood remains in the centre with one or more concentric rings of clearer fluid developing around it. Bruising in the mastoid region (Battle's sign) also indicates basal skull fracture, but the bruising usually takes 12 to 36 hours to develop. Blood seen behind the tympanic membrane (haemotympanum) may also indicate a basal skull fracture. Fractures through the cribriform plate are frequently associated with bilateral periorbital haematomas. Subconjunctival haematoma may occur from direct orbital roof fracture, in which case there is no posterior limit to the haematoma. All these signs may take several hours to develop and may not be present in a casualty seen immediately after injury. Basal fractures are very difficult to diagnose from plain X-ray films. If X-ray facilities are available you should look for the presence of intracranial air or bleeding into anatomically related air sinuses.

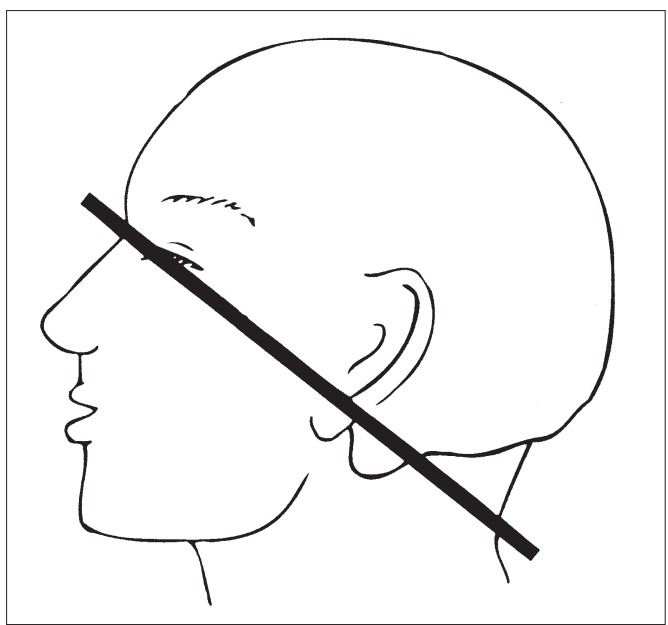

Fig 8.3 Diagonal line showing the level of the base of the skull.

\section{Triage of Head Injuries}

Which casualties should be evacuated? 0835. In peacetime practice, head injury management guidelines are usually agreed between local A\&E and neurosurgical centres. In ideal circumstances: 
a. Severe head injuries need neurosurgical referral:

- Coma score (GCS) less than 8.

- Compound or depressed skull fracture.

- Skull fracture with neurological signs.

- Fractured base of skull.

b. Moderate head injuries should be admitted to hospital for further investigation and observation including CT scanning:

- Simple skull fracture.

- Neurological signs (including confusion).

- Casualty is difficult to assess because of drugs or alcohol.

c. Minor head injuries following the loss of consciousness need 24 hours observation, done by designated personnel. These casualties are:

- Fully orientated.

- No skull fracture.

- No neurological signs.

d. Should the casualty develop any of the following, then they need to be assessed by a medical officer with a view to hospital admission:

- Vomiting.

- Drowsiness.

- Fits.

- Double/blurred vision.
0836. In less than ideal circumstances on the battlefield, the task is to identify those casualties who will benefit from evacuation to a neurosurgical unit and those whose outlook is so poor that they should not be put into the evacuation chain ahead of priority. This decision must be made on the basis of clinical findings. In short, casualties who require emergency surgery are those with depressed or open skull fractures and those with focal lesions causing symptoms of raised intracranial pressure. The head injury triage scheme at Table 8-2 is not foolproof but will act as a guide.

\section{Summary}

Remember the $A B C D E$ routine.

- Prevent secondary injury by:

- Preventing hypoxia, hypercarbia and hypovolaemia.

- Giving oxygen if available and ensure a clear airway at all times.

- Treating fits with diazepam in appropriate doses.

- Establishing a working diagnosis.

- Searching for associated injuries.

- Constantly repeating the mini-neurological examination.

- Identifying and evacuating appropriate casualties to a neurosurgical unit. 


\section{Glasgow Coma Scale Score}

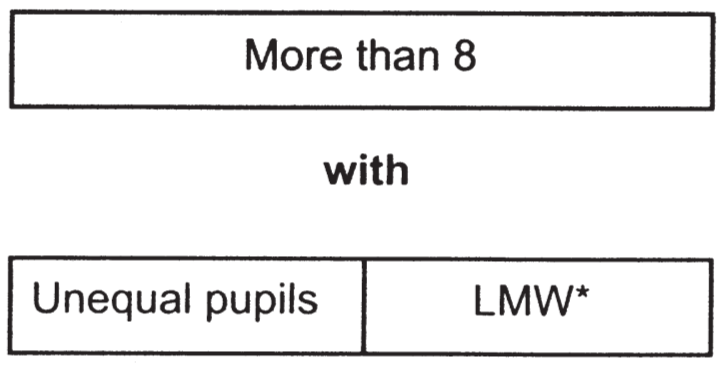

and/or

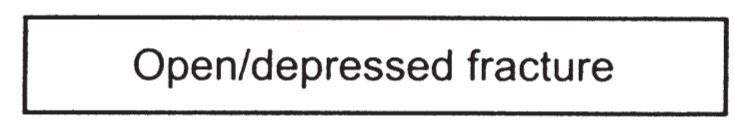

and/or

? Basal skull fracture

and/or

Foreign body penetration

and/or

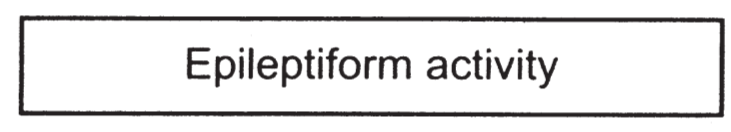

and/or

Hyperthermia

and/or

Fall in GCS score

Evacuate all these as urgently as the situation allows

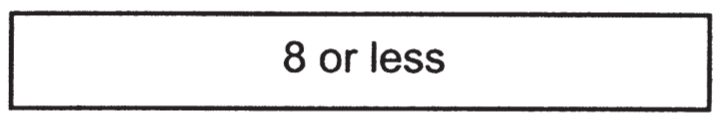

with or without
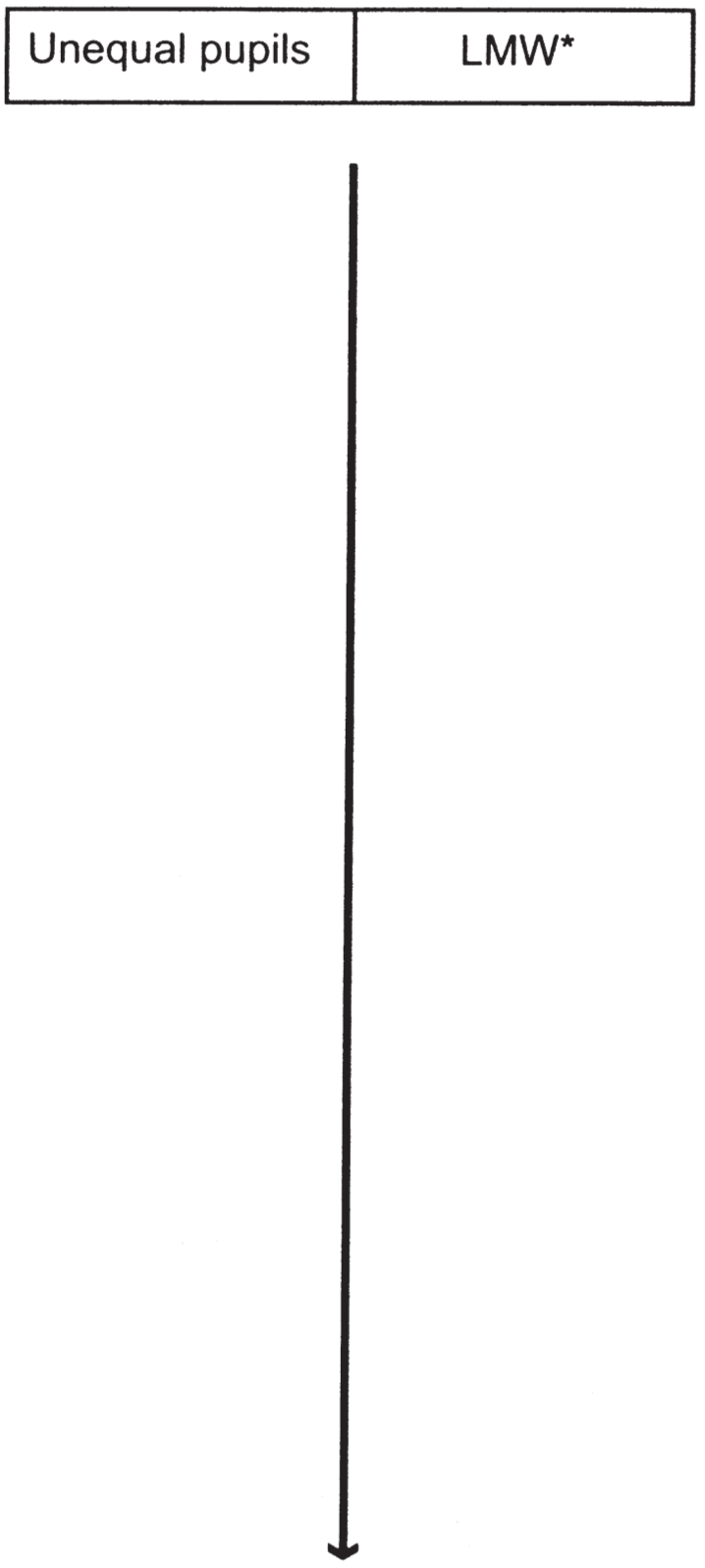

Evacuate these as low priority depending on casualty loading and tactical situation 